\title{
Energy Storage for Energy Security
}

\author{
Søren Linderoth \\ Technical University of Denmark, Denmark \\ Sqli@dtu.dk
}

The tendency is that energy production increasingly is coming from renewable sources. Reasons behind are several, but primarily in order to reduce the green-house effect and global warming due to the use of fossil fuels. The renewables to replace the fossil fuels will be from wind, solar and biomass. The cost of wind and solar plants are now at compelling prices compared to coal fired plants, even without taking into account the externalities, such as cost of pollution. However, in contrast to the existing use of fossil fuels, the power production from wind and solar is fluctuating, and production is not correlated to the consumption. This can in part be taken care of by trading of electricity between regions and countries. For example, Denmark is at all times exchanging electricity with the neighboring countries Norway, Sweden and Germany to keep the balance. However, this will challenged in the future, because the amount of wind energy, in all the countries, are increasing significantly. Today, about $7 \%$ of the energy consumption in Denmark is coming from wind - in the future, this number will be much larger for Denmark to become independent on fossil fuels. To secure that power and energy can be provided when needed or wanted, energy and power reservoirs must be in place. The reservoirs can be of small, medium and of very large sizes, they can be for instant power supply, or they can be for medium to long-term supply for days and weeks. In this presentation technologies being developed for medium- to large-scale energy storage of electricity will be presented and discussed. Possible gas-flow and liquid-flow type batteries can be future storage technologies, e.g. reversible solid oxide cell batteries integrated with thermal storage would one option to be able to provide large energy storage potential. Organic flow batteries are other possible storage technologies being developed. Conversion of electricity from wind and solar, combined with carbon from biomass or $\mathrm{CO} 2$ exhaust, into fuels and chemicals (Power2X) will be important for replacing fossil supply in some of the manufacture industry, and can be essential for parts of the transport sector, while also be a storage media. An externality of renewables is the need of storage, hence the cost reduction is one strong driving force for the research in energy storage technologies. 